\title{
ABO Blood Group, Epstein-Barr virus Infection and Prognosis of Patients with Non-metastatic Nasopharyngeal Carcinoma
}

\author{
Ya-Xiong Zhang, 1,2,3,4 , Shi-Yang Kang1,2,3,4\&, Gang Chen ${ }^{1,2,3,4 \&}$, Wen-Feng Fang1,2,3, \\ Xuan Wu ${ }^{1,2,3}$, Hua-Jing You ${ }^{1,2,3,4}$, Da-Cheng He $\mathrm{He}^{1,2,3,4}$, Ya-Lin Cao ${ }^{1,2,3,4}$, Wen-Hua \\ Liang $^{1,2,3 *}$, Li Zhang ${ }^{1,2,3 *}$
}

\begin{abstract}
Background: A prior study showed blood type $\mathrm{A} / \mathrm{AB}$ to be associated with an increased risk of nasopharyngeal carcinoma (NPC) compared to subjects with blood type O. However, the relationship between ABO blood groups and prognosis of NPC patients is still questionable. In addition, whether Epstein-Barr virus (EBV) infection is associated with prognosis of NPC patients with different $\mathrm{ABO}$ blood groups is unclear. Materials and Methods: We conducted univariate and multivariable Cox regression analyses based on a consecutive cohort of 1,601 patients to investigate the above issues. Results: There was no significant difference in overall survival (OS) between different ABO blood groups $(p=0.629)$, neither between A vs. non-A blood groups $(p=0.895)$ nor AB vs. non-AB blood group $(p=0.309)$ in univariate analyses and after adjusting for other factors. Interaction tests revealed that high immunoglobulin A against Epstein-Barr virus viral capsid antigen (VcA-IgA) level was associated with a favorable prognosis in male patients with UICC stage II disease who had an A blood type $(p=0.008)$, compared with those with non-A blood type. In addition, male patients with an A blood group with a high blood lymphocyte level showeda tendency towards better survival in UICC stage III $(p=0.096)$. Conclusions: ABO blood group status is not associated with the prognosis of patients with NPC. Additionally, blood group A male NPC patients with high VcA-IgA level or high blood lymphocyte counts might be correlated with a favorable prognosis in UICC stage II or III, respectively.
\end{abstract}

Keywords: Nasopharyngeal carcinoma - ABO blood group - Epstein-Barr virus - VcA-IgA - blood lymphocyte

Asian Pac J Cancer Prev, 15 (17), 7459-7465

\section{Introduction}

Nasopharyngeal carcinoma (NPC) is a common head and neck malignant neoplasm, especially in southern China where the major pathological type of NPC is nonkeratinizing undifferentiated carcinoma (World Health Organization Type III) (Jemal et al., 2011; Huang et al., 2012). Several factors have been proved to enhance the risk of NPC, including Epstein-Barr virus (EBV), smoking, alcohol consumption, salt-preserved foods and family history of cancer, etc (Chien et al., 2001; Jia et al., 2010; Ji et al., 2011; Hashim et al., 2012).

Extensive investigations have showed that the $\mathrm{ABO}$ blood group might be associated with risk to certain malignancies (Iodice et al., 2010; Gates et al., 2011; Joh et al., 2012; Wang et al., 2012; Zhang et al., 2014). A previous study suggested that blood type A or AB was associated with an increased risk of NPC and male patients with blood group A had higher rates of distant metastasis than those in other blood groups, while the prognostic value of blood group A remained uncertain (Sheng et al., 2013). Another study reported that NPC patients of WHO histological type III with A blood group had significantly lower overall survival (OS) than those with non-A blood group (Ouyang et al., 2013). To our knowledge, however, we still lack substantial evidence to determine the relationship between $\mathrm{ABO}$ blood group and prognosis in NPC. Besides, it remains unknown that whether EBV infection, which is highly correlated with the etiology and development of endemic NPC, has an impact on this relationship.

Therefore, we sought to conduct a study to assess the prognostic value of different blood groups in southern Chinese NPC patients. In addition, we evaluated whether EBV infection in different blood groups might be associated with the prognosis of a large cohort of NPC patients, especially in those male patients with an Ablood group. 


\section{Materials and Methods}

\section{Study Population}

1601 patients with non-metastatic NPC who were newly diagnosed during November 2000 to September 2003 at Sun Yat-Sen University Cancer Center (Guangzhou, China) were included in the study. This study was approved by the Institutional Review Board of Sun YatSen University Cancer Center (Guangzhou, China). All the patients had provided written informed consent. All of the records such as clinical and pathological features were collected before blood sampling with no patients having received any treatment, for instance radiotherapy or chemotherapy. The clinicopathological features of the patients included gender, age, smoking history, drinking history, UICC stage, tumor size (T category), lymph node status ( $\mathrm{N}$ category), pathological type (WHO type), serum immunoglobulin A against Epstein-Barr virus viral capsid antigen (VcA-IgA) level, serum immunoglobulin A against Epstein-Barr virus viral early antigen (EA-IgA) level, peripheral blood lymphocyte counts, radiotherapy, chemotherapy and ABO blood group. All patients were restaged by seventh edition of UICC Staging System for NPC.

Categories of each characteristic were divided as following: for age, patients more than 50 years old were considered as the older group. Smoking and alcohol history were noted as yes or no. Low VcA-IgA level was defined, if the titer was $\leq 1: 320$. Similarly, EA-IgA was considered as negative when the titer was 0 . Low

A

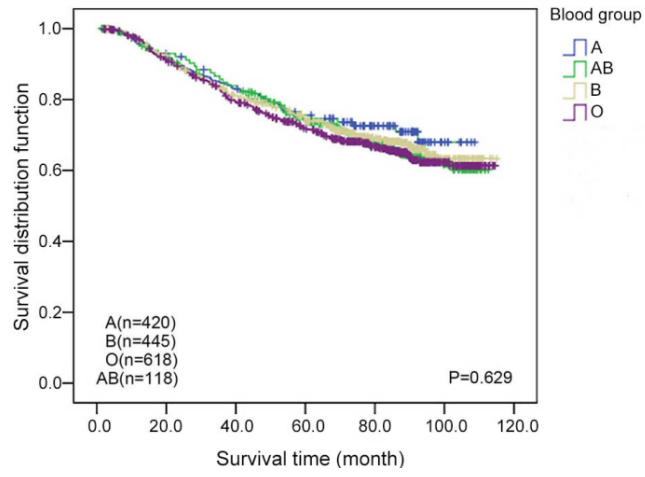

B

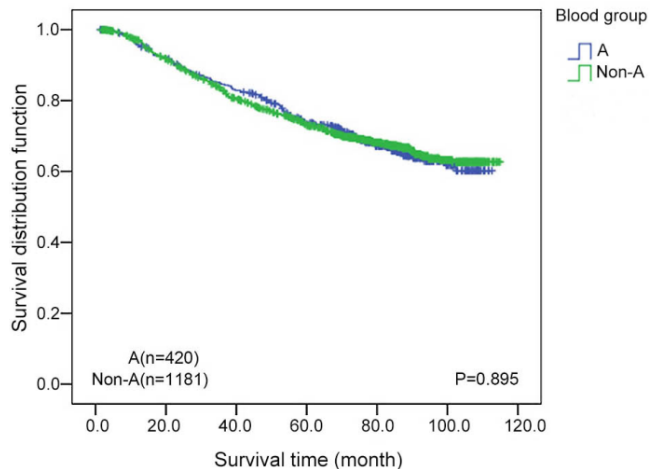

Figure 1. Overall Survival in Patients with Nasopharyngeal Carcinoma Stratified for ABO Blood Group Status (A) and Stratified for A Blood Group Status (B) serologic lymphocytes level was defined, if the titer was $\leq 1.35 \times 10^{9} / \mathrm{L}$. Radiotherapy types included none, conventional radiotherapy (CRT), computed tomographysimulation (CT-SIM), 3-dimensioned conventional radiotherapy (3D-CRT) and intensity-modulated radiotherapy (IMRT). While chemotherapy patterns were none, induction chemotherapy (IC), concomitant chemotherapy (CC), IC+CC. Follow-up visits were scheduled every 2 months after treatment at SYSU Cancer Center. The follow-up duration was estimated from the first day of treatment to either the day of death or the day of last examination.

\section{Statistical analyses}

SPSS 17.0 software was used for the statistical analysis. Continuous variables were divided into different categories as mentioned above. All the cut-off values were obtained by X-tile software (Version 3.6.1, Yale University, New Haven, CT), taking clinical expertise into consideration. The OS analyses were estimated with Kaplan-Meier method and log-rank test to assess the possible individual risk factors related with survival. Further investigations of multivariable analyses were performed by Cox regression for factors which were significantly associated in univariate survival analyses. Results were reported with hazard ratio (HR), corresponding $95 \%$ confidence intervals (CI). A $p$-value $<0.05$ was considered statistically significant.

\section{A}

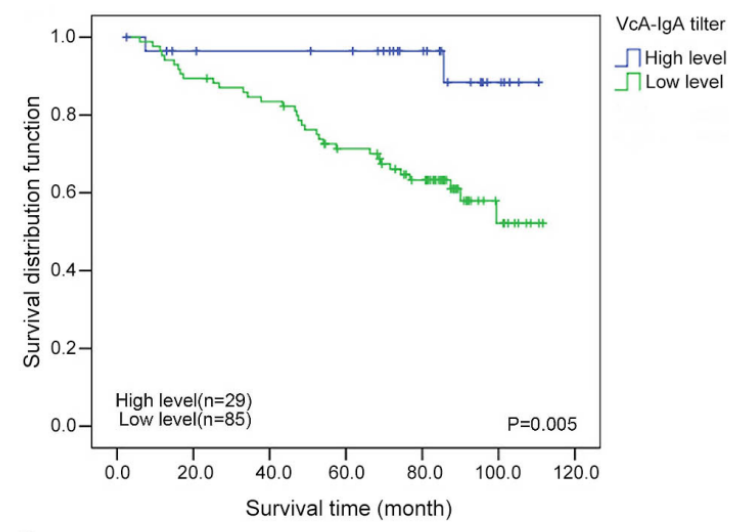

B

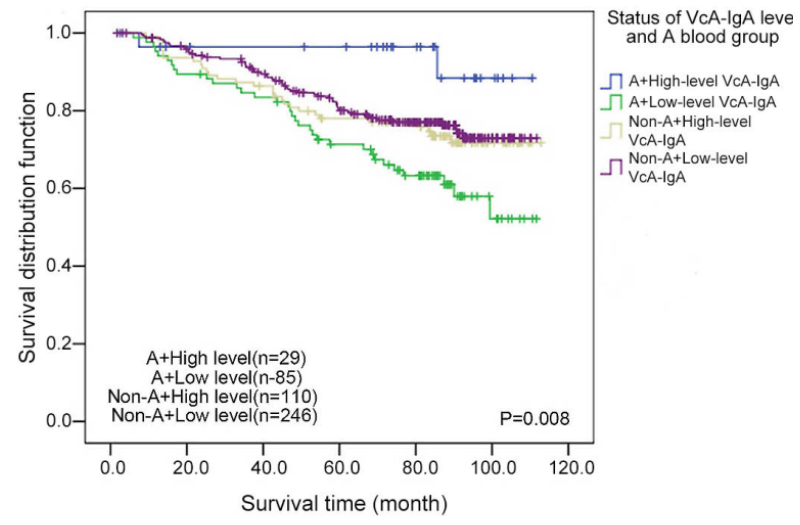

Figure 2. Overall Survival in Male Nasopharyngeal Carcinoma Patients with A Blood Group of UICC Stage II Stratified for Serum VcA-IgA Level (A) and Stratified for Serum VcA-IgA Level and A Blood Group Status (B) 
ABO Blood Group, Epstein-Barr virus Infection and Prognosis of Patients with Non-metastatic Nasopharyngeal Carcinoma

\section{Results}

Clinicopathological characteristics of the patients

A total of 1601 NPC patients with known ABO blood group were included in this study. All patients enrolled were non-metastatic cases with $77.3 \%$ male and $22.7 \%$ female ones. The proportion of blood groups A, B, O and $\mathrm{AB}$ were $25.55 \%, 27.81 \%, 38.72 \%, 7.92 \%$ in male, and $28.57 \%, 27.75 \%, 38.14 \%, 5.49 \%$ in female, respectively. There was no difference in the distribution of basic characteristics among different blood types (all $p$ value $\geq 0.05$ ). Half of patients $(47.78 \%)$ were in UICC I or II stage. As for pathological types, cases were predominantly WHO type III. The Median follow-up time was 85.72 months. Clinicopathological features of the patients were presented in Table 1.

ABO blood group status is not associated with the prognosis of patients with NPC

There was no statistically difference in OS among the different $\mathrm{ABO}$ blood groups in univariate analyses $(p=0.629)$ (Figure 1A). Similarly, no statistical significance was found between patients of $A$ and non-A blood groups $(p=0.895)$ (Figure 1B), as well as AB and non-AB blood

Table 1. Clinicopathologic Characteristics of 1601 Patients with Nasopharyngeal Carcinoma

\begin{tabular}{|c|c|c|c|c|c|c|}
\hline Parameter & Total & Blood group A & Blood group B & Blood group $\mathrm{O}$ & Blood group AB & $p$ value \\
\hline 1601 & 420 & 445 & 618 & 118 & & \\
\hline Gender & & & & & 0.354 & \\
\hline Male & $1237(77.3 \%)$ & $316(75.2 \%)$ & $344(77.3 \%)$ & $479(77.5 \%)$ & $98(83.1 \%)$ & \\
\hline Female & $364(22.7 \%)$ & $104(24.8 \%)$ & $101(22.7 \%)$ & $139(22.5 \%)$ & $20(16.9 \%)$ & \\
\hline Age[years] & & & & & & 0.623 \\
\hline$\leq 50$ & $1019(63.6 \%)$ & $271(64.5 \%)$ & $273(61.3 \%)$ & $396(64.1 \%)$ & $79(66.9 \%)$ & \\
\hline$>50$ & $582(36.4 \%)$ & $149(35.5 \%)$ & $172(38.7 \%)$ & $222(35.9 \%)$ & $39(33.1 \%)$ & \\
\hline Smoking status & & & & & & 0.262 \\
\hline Yes & $791(49.4 \%)$ & $195(46.4 \%)$ & $227(51.0 \%)$ & $303(49.0 \%)$ & $66(55.9 \%)$ & \\
\hline No & $810(50.6 \%)$ & $225(53.6 \%)$ & $218(49.0 \%)$ & $315(51.0 \%)$ & $52(44.1 \%)$ & \\
\hline Drinking status & & & & & & 0.104 \\
\hline Yes & $248(15.5 \%)$ & $68(16.2 \%)$ & $66(14.8 \%)$ & $87(14.1 \%)$ & $27(22.9 \%)$ & \\
\hline No & $1353(84.5 \%)$ & $352(83.8 \%)$ & $379(85.2 \%)$ & $531(85.9 \%)$ & $91(77.1 \%)$ & \\
\hline UICC stage & & & & & & 0.59 \\
\hline I & $86(5.4 \%)$ & $26(6.2 \%)$ & $24(5.4 \%)$ & $30(4.9 \%)$ & $6(5.1 \%)$ & \\
\hline II & $679(42.4 \%)$ & $173(41.2 \%)$ & $190(42.7 \%)$ & $266(43.0 \%)$ & $50(42.4 \%)$ & \\
\hline III & $543(33.9 \%)$ & $134(31.9 \%)$ & $148(33.3 \%)$ & $213(34.5 \%)$ & $48(40.7 \%)$ & \\
\hline IV & $293(18.3 \%)$ & $87(20.7 \%)$ & $83(18.7 \%)$ & $109(17.6 \%)$ & $14(11.9 \%)$ & \\
\hline T category & & & & & & 0.141 \\
\hline $\mathrm{T} 1$ & $282(17.6 \%)$ & $77(18.3 \%)$ & $85(19.1 \%)$ & $102(16.5 \%)$ & $18(15.3 \%)$ & \\
\hline $\mathrm{T} 2$ & $781(48.8 \%)$ & $205(48.8 \%)$ & $215(48.3 \%)$ & $295(47.7 \%)$ & $66(55.9 \%)$ & \\
\hline $\mathrm{T} 3$ & $284(17.7 \%)$ & $60(14.3 \%)$ & $74(16.6 \%)$ & $129(20.9 \%)$ & $21(17.8 \%)$ & \\
\hline $\mathrm{T} 4$ & $254(15.9 \%)$ & $78(18.6 \%)$ & $71(16.0 \%)$ & $92(14.9 \%)$ & $13(11.0 \%)$ & \\
\hline $\mathrm{N}$ category & & & & & & 0.788 \\
\hline NO & $445(27.8 \%)$ & $115(27.4 \%)$ & $123(27.6 \%)$ & $175(28.3 \%)$ & $32(27.1 \%)$ & \\
\hline N1 & $692(43.2 \%)$ & $185(44.0 \%)$ & $192(43.1 \%)$ & $271(43.9 \%)$ & $44(37.3 \%)$ & \\
\hline $\mathrm{N} 2$ & $417(26.0 \%)$ & $109(26.0 \%)$ & $116(26.1 \%)$ & $152(24.6 \%)$ & $40(33.9 \%)$ & \\
\hline N3 & $47(2.9 \%)$ & $11(2.6 \%)$ & $14(3.1 \%)$ & $20(3.2 \%)$ & $2(1.7 \%)$ & \\
\hline Pathological Type & & & & & & 0.107 \\
\hline WHO II & $115(7.2 \%)$ & $26(6.2 \%)$ & $43(9.7 \%)$ & $37(6.0 \%)$ & $9(7.6 \%)$ & \\
\hline WHO III & $1486(92.8 \%)$ & $109(92.4 \%)$ & $581(94.0 \%)$ & $402(90.3 \%)$ & $394(93.8 \%)$ & \\
\hline VcA-IgA & & & & & & 0.102 \\
\hline High level & $534(34.2 \%)$ & $120(29.2 \%)$ & $157(36.2 \%)$ & $216(35.9 \%)$ & $41(35.3 \%)$ & \\
\hline $\begin{array}{l}\text { Low level } \\
\text { missing values }\end{array}$ & $\begin{array}{c}1028(65.8 \%) \\
39\end{array}$ & $291(70.8 \%)$ & $277(63.8 \%)$ & $385(64.1 \%)$ & $75(64.7 \%)$ & \\
\hline EA-IgA & & & & & 0.565 & \\
\hline Positive & $1218(78.6 \%)$ & $313(76.9 \%)$ & $342(79.5 \%)$ & $468(78.4 \%)$ & $95(82.6 \%)$ & \\
\hline Negative & $331(21.4 \%)$ & $94(23.1 \%)$ & $88(20.5 \%)$ & $129(21.6 \%)$ & $20(17.4 \%)$ & \\
\hline Lymphocyte counts cocounts & & & & & & 0.377 \\
\hline High level & $1283(80.1 \%)$ & $344(81.9 \%)$ & $346(77.8 \%)$ & $501(81.1 \%)$ & $92(78.0 \%)$ & \\
\hline Low level & $318(19.8 \%)$ & $76(18.1 \%)$ & $99(22.2 \%)$ & $117(18.9 \%)$ & $26(22.0 \%)$ & \\
\hline Radiotherapy & & & & & & 0.773 \\
\hline None & $12(0.7 \%)$ & $4(1.0 \%)$ & $5(1.1 \%)$ & $3(0.5 \%)$ & $0(0.0 \%)$ & \\
\hline CRT & $1354(84.6 \%)$ & $345(82.1 \%)$ & $377(84.7 \%)$ & $531(85.9 \%)$ & $101(85.6 \%)$ & \\
\hline CT-SIM & $171(10.7 \%)$ & $52(12.4 \%)$ & $49(11.0 \%)$ & $58(9.4 \%)$ & $12(10.2 \%)$ & \\
\hline 3D-CRT & $33(2.1 \%)$ & $8(1.9 \%)$ & $9(2.0 \%)$ & $14(2.3 \%)$ & $2(1.7 \%)$ & \\
\hline IMRT & $31(1.9 \%)$ & $11(2.6 \%)$ & $5(1.1 \%)$ & $12(1.9 \%)$ & $3(2.5 \%)$ & \\
\hline Chemotherapy & & & & & & 0.654 \\
\hline None & $824(51.5 \%)$ & $218(51.9 \%)$ & $220(49.4 \%)$ & $326(52.8 \%)$ & $60(50.8 \%)$ & \\
\hline $\mathrm{IC}$ & $417(26.0 \%)$ & $116(27.6 \%)$ & $125(28.1 \%)$ & $146(23.6 \%)$ & $30(25.4 \%)$ & \\
\hline $\mathrm{CC}$ & $229(14.3 \%)$ & $56(13.3 \%)$ & $61(13.7 \%)$ & $97(15.7 \%)$ & $15(12.7 \%)$ & \\
\hline $\mathrm{IC}+\mathrm{CC}$ & $131(8.2 \%)$ & $30(7.1 \%)$ & $39(8.8 \%)$ & $49(7.9 \%)$ & $13(11.0 \%)$ & \\
\hline
\end{tabular}


Table 2. Univariate and multivariate analyses of overall survival in nasopharyngeal carcinoma patients*

\begin{tabular}{|c|c|c|c|c|c|c|}
\hline \multirow[t]{2}{*}{ Statistic index } & \multicolumn{3}{|c|}{ Blood group status } & \multicolumn{3}{|c|}{ Blood group A status } \\
\hline & $A(n=407)$ & $B(n=429)$ & $O(n=597)$ & $\mathrm{AB}(\mathrm{n}=115)$ & $A(n=407)$ & Non-A $(n=1141)$ \\
\hline No. of events & 134 & 130 & 199 & 32 & 134 & 361 \\
\hline uHR $(95 \%$ CI $)$ & 1 & $0.915(0.719-1.164)$ & $1.045(0.839-1.301)$ & $0.851(0.579-1.252)$ & 1 & $0.975(0.800-1.189)$ \\
\hline P-value & & 0.468 & 0.695 & 0.413 & & 0.804 \\
\hline aHR (95\% CI)a & 1 & $0.896(0.702-1.143)$ & $1.081(0.865-1.351)$ & $0.843(0.572-1.244)$ & 1 & $0.982(0.803-1.200)$ \\
\hline P-value & & 0.378 & 0.491 & 0.39 & & 0.856 \\
\hline
\end{tabular}

*A total of 53 patients with missing values were excluded (Likelihood-Ratio Test: $p<0.0001)$. $* *$ Abbreviations: NPC $=$ nasopharyngeal carcinoma; uHR $=$ unadjusted hazard ratio; $\mathrm{CI}=$ confidence interval; aHR = adjusted hazard ratio; UICC $=$ Union for International Cancer Control; $\mathrm{T}$ category $=$ tumor size; $\mathrm{N}$ category $=$ lymph node status; VcA-IgA = immunoglobulin A against Epstein-Barr virus viral capsid antigen; EA-IgA = immunoglobulin A against Epstein-Barr virus viral early antigen; CRT = conventional radiotherapy; CT-SIM = computed tomography-simulation; 3D-CRT = 3-dimensioned conventional radiotherapy; IMRT = intensity-modulated radiotherapy; $\mathrm{IC}=$ induction chemotherapy; $\mathrm{CC}=$ concomitant chemotherapy. aAdjusted for gender, age ( $\leq 50 \mathrm{vs.}>50)$, smoking history (yes vs. no), drinking history (yes vs. no), UICC stage (I/ II/ III/ IV), T category (T1/ T2/ T3/ T4), N category (N0/ N1/ N2/ N3), pathological type (WHO type II / III), VcA-IgA titer ( 1 1:320 vs. > 1:320), EAIgA titer (negative vs. positive), peripheral blood lymphocyte counts $\left(\leq 1.35 \times 10^{\circ} / \mathrm{L} v s .>1.35 \times 10^{\circ} / \mathrm{L}\right)$, radiotherapy type (none, CRT, CT-SIM, 3D-CRT, and IMRT), and chemotherapy type (none, IC, CC, and IC + CC).

Table 3. Univariate and Multivariate Analyses of Overall Survival in WHO Type III Nasopharyngeal Carcinoma Patients with Chemotherapy in Conventional Radiotherapy Cohort ${ }^{\#}$

\begin{tabular}{|c|c|c|c|c|c|c|}
\hline \multirow[t]{2}{*}{ Statistic index } & \multicolumn{3}{|c|}{ Blood group status } & \multicolumn{3}{|c|}{ Blood group A status } \\
\hline & $A(n=152)$ & $B(n=166)$ & $O(n=221)$ & $\mathrm{AB}(\mathrm{n}=48)$ & $\mathrm{A}(\mathrm{n}=152)$ & Non-A $(n=435)$ \\
\hline No. of events & 57 & 65 & 94 & 17 & 57 & 176 \\
\hline uHR (95\% CI) & 1 & $1.083(0.759-1.545)$ & $1.245(0.896-1.730)$ & $0.952(0.554-1.637)$ & 1 & $1.147(0.851-1.547)$ \\
\hline P-value & & 0.661 & 0.192 & 0.859 & & 0.367 \\
\hline aHR $(95 \%$ CI)a & 1 & $1.037(0.724-1.487)$ & $1.275(0.910-1.786)$ & $0.930(0.536-1.615)$ & 1 & $1.136(0.840-1.537)$ \\
\hline P-value & & 0.842 & 0.157 & 0.798 & & 0.408 \\
\hline
\end{tabular}

*\#A total of 15 patients with missing values were excluded (Likelihood-Ratio Test: $p<0.0001$ ). Abbreviations: NPC=nasopharyngeal carcinoma; uHR=unadjusted hazard ratio; $\mathrm{CI}=$ confidence interval; aHR=adjusted hazard ratio; UICC=Union for International Cancer Control; $\mathrm{T}$ category = tumor size; $\mathrm{N}$ category=lymph node status; VcAIgA=immunoglobulin A against Epstein-Barr virus viral capsid antigen; EA-IgA=immunoglobulin A against Epstein-Barr virus viral early antigen .

aAdjusted for gender, age ( $\leq 50$ vs. $>50$ ), smoking history (yes vs. no), drinking history(yes vs. no), UICC stage (I/ II/ III/ IV), T category (T1/ T2/ T3/ T4), N category (N0/ N1/ N2/ N3), VcA-IgA titer ( $\leq 1: 320$ vs. $>1: 320$ ), EA-IgA titer (negative vs. positive), peripheral blood lymphocyte counts $\left(\leq 1.35 \times 10^{9} / \mathrm{L}\right.$ vs. $\left.>1.35 \times 10^{9} / \mathrm{L}\right)$.

groups ( $p=0.309)$. Through multivariate analyses, we used blood group A as reference category and confirmed that there was no significant survival benefit for patients with blood group B (HR,0.896; 95\%CI,0.702-1.143; $p=0.378)$, O (HR,1.081; 95\%CI, 0.865-1.351; $p=0.491)$ and AB (HR,0.843; 95\%CI, 0.572-1.244; $p=0.390)$, as well as those with non-A blood groups (HR ,0.982; 95\%CI,0.803$1.200 ; p=0.856$ ) (Table 2).

We failed to detect statistically significant association between $\mathrm{OS}$ and $\mathrm{ABO}$ group either $(\mathrm{HRB} / \mathrm{A}=1.037$, 95\%CI, 0.724-1.487, $p=0.842 ; \mathrm{HRO} / \mathrm{A}=1.275,95 \% \mathrm{CI}$, $0.910-1.786, p=0.157$; HRAB/A=0.930, 95\%CI, 0.536$1.615, p=0.798$ ), when focusing on WHO type III patients in CRT cohort with chemotherapy history in multivariate Cox Regression analyses. In addition, we obtained similar results in terms of A vs. non-A blood groups (HRNonA/A,1.136; 95\%CI,0.840-1.537; $p=0.408$ ) (Table 3).

Potential protective effect of VcA-IgA in male NPC patients with A blood group in UICC stage II

We assessed the prognostic value of serum VcA-IgA level in male patients of blood group A within the same UICC stage. In univariate analysis, high VcA-IgA level was associated with a favorable prognosis only in UICC stage II $(p=0.005)$ (Figure 2A), whereas no significant difference was observed in OS between high and low VcAIgA level respectively in UICC stage III and IV ( $p=0.737$; $p=0.236$ ), and there was no male NPC patient of blood group A with high serum VcA-IgA level in UICC stage I.

In order to assess the interaction effect, male patients of UICC stage II were divided into four subgroups according to blood group status (A vs. non-A) and VcA-
A

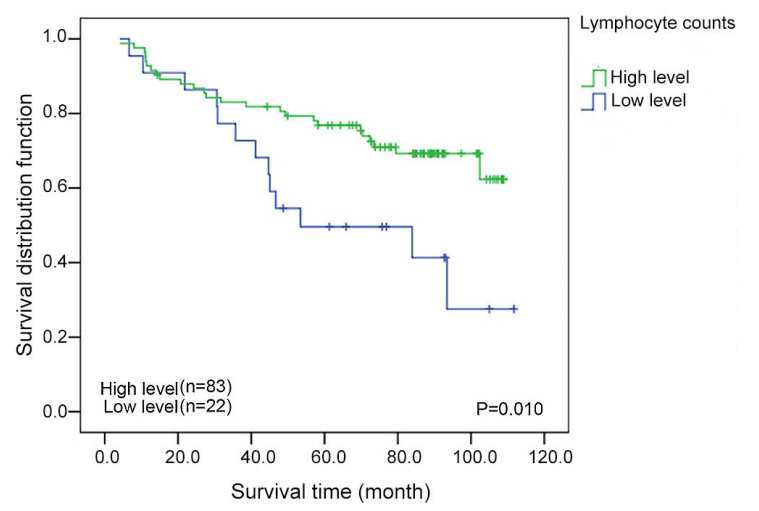

B

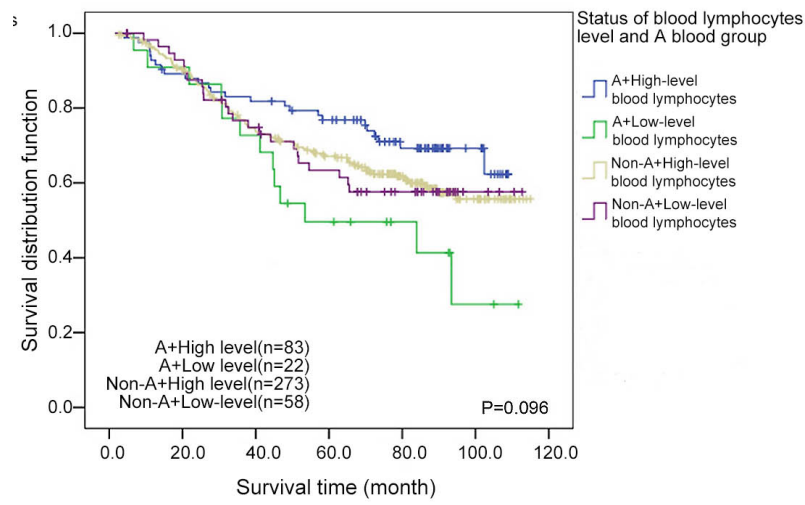

Figure 3. Overall Survival in Male Nasopharyngeal Carcinoma Patients with A Blood Group of UICC Stage III Stratified for Peripheral Blood Lymphocyte Counts (A) and Stratified for Peripheral Blood Lymphocyte Counts and A Blood Group Status (B) 
IgA level (low level vs. high level). It was revealed that there was an OS advantage for male patients of blood group A with high VcA-IgA level compared to those with low level, whereas there was no significant difference of OS between non-A groups ( $p=0.008$ ) (Figure $2 \mathrm{~B}$ ). When female patients of UICC stage II were divided into four subgroups as above, there was no significant difference of OS among them $(p=0.165)$.

Potential protective effects of peripheral blood lymphocyte in male NPC patients with A blood group in UICC stage III

We also sought to find out the prognostic value of peripheral blood lymphocyte counts in male patients of blood group A within the same UICC stage. Patients with high blood lymphocyte level had a favorable prognosis only in UICC stage III ( $p=0.010)$ in univariate analyses (Figure 3A), whereas there was no significant difference in OS in other UICC stages respectively (I: $p=0.482$; II: $p=0.877$; IV: $p=0.698$ ).

Besides, male patients of UICC stage III were categorized into four subgroups according to blood group status (A vs. non-A) and blood lymphocyte level (low level vs. high level). Interaction test revealed similar trends, whereas there was no significant difference of OS between non-A groups ( $p=0.096$ ) (Figure 3B). When female patients of UICC stage III were divided into four subgroups as above, there was no significant difference of OS among them $(p=0.523)$.

\section{Discussion}

The ABO blood group system was first discovered by Karl Landsteiner, who found three different blood types (A, B, and O) in 1900. Among 29 blood group systems, ABO blood group system is the most valuable one (Hosoi, 2008). ABO blood group genes are mapped at the chromosome $9 \mathrm{q} 34.1$ to $9 \mathrm{q} 34.2$ (Ozelius et al., 1992). Links between ABO blood groups with benign or malignant diseases, such as gastric and pancreas cancers, have been observed for a long time (Wang et al., 2012; Wolpin et al., 2009). It was proved that ABO blood group antigen expression on cancer cells was modified by hypermethylation of ABO gene promoter (Gao et al., 2004), which might be related with the invasiveness or metastasis of tumor. A latest research suggested that blood type A or AB was associated with an increased risk of NPC compared with subjects with blood type O (Sheng et al., 2013). In addition, NPC patients of WHO histological type III with A blood group had significantly lower OS than those with non-A blood group (Ouyang et al., 2013).

Based on these investigations, we hypothesized that the genetic background with $\mathrm{A}$ or $\mathrm{AB}$ blood group might predispose those patients who actually developed NPC to a more favorable prognosis. In our study, however, there was no statistically significant difference among different ABO blood groups regardless of assortment. Moreover, when we only selected WHO type III patients in CRT cohort with chemotherapy history to imitate similar enrollment of the previous study (Ouyang et al., 2013), it still showed no significant difference between A and non-A blood group in terms of OS. Notably, there was basically no overlap on the selection of NPC patients between this study (year 2000-2003) and the prior study (Ouyang et al., 2013) (year 2003-2006). The reason why conflicting results were obtained from different periods is worthy of further research.

Therefore, we concluded that ABO blood group status was not associated with the prognosis of patients with NPC. It is still questionable whether the OS of A blood group patients with WHO histological type III is significantly lower than that of non-A blood group ones.

NPC in the epidemic area is special because of its strong association with EBV and sustain higher levels of serum EBV-specific antibodies, especially isotypes of immunoglobulin A (IgA) (Henle et al., 1976; Ho et al., 1976; Han et al., 2012). Previous studies have shown that $\mathrm{VcA}-\operatorname{IgA}$ and EA-IgA were particularly elevated in NPC patients (Sam et al., 1989; Low et al., 2000; Cheng et al., 2002; Tiwawech et al., 2003) and might provide information regarding the prognosis (Cai et al., 2013).

We observed that the HR-value of VcA-IgA was below 1 while the HR-value of EA-IgA was above 1 in multivariate analyses after adjusting for UICC stage. It suggested that a false poor prognosis of high VcA-IgA level was possibly due to the confounding effects of tumor burden (Fan H et al., 2004). But actually, VcA-IgA might show protective effects in a certain UICC stage. Therefore, we tested the protective effect of VcA-IgA according to different criteria of classification, such as gender, UICC stage, blood types and so on. Finally, the interaction test revealed that there was an OS advantage for male patients of blood group A with high $\mathrm{VcA}-\operatorname{Ig} \mathrm{A}$ level within stage II compared with those with low level, whereas there was no significant difference of OS between non-A subgroups. Interestingly, a recent study showed the rate of distant metastasis was significantly higher among male patients with A blood group than that of non-A blood group (Sheng et al., 2013), which indicated that male NPC patients with blood group A were prone to metastasize distantly. It was possible that high VcA-IgA level might inhibit tumor metastasis and bring benefit to the prognosis, as a protective antibody. We assumed that VcA-IgA might play a role in suppression of metastasis of NPC cells which might express virus-related antigens on the surface, although more direct evidences are needed to verify this hypothesis.

However, no significant difference of OS respectively in UICC stage III and IV was detected, which might due to the restriction of small sample size. The protective effect of VcA-IgA was likely limited, thereby being masked by larger tumor burden in stage III and IV. It was worth mentioning that there was no male NPC patient of blood group A with high serum VcA-IgA level in UICC stage I, which inferred that high serum VcA-IgA level possesses potential protective effect in male patients of blood group A with NPC in early UICC stage.

NPC is also denominated lymphoepithelioma because of its intensive infiltration of lymphocytes among EBVinfected epithelial malignant cells (Rickinson et al., 2007). Because high-level VcA-IgA in male NPC patients of blood group A was associated with a favorable prognosis in UICC stage II, we tried to investigate whether the index 
of cellular immunity, such as peripheral blood lymphocyte counts, was associated with better prognosis in certain clinical stage.

The interaction test revealed similar tendency that the OS of male NPC patients of A blood group in UICC stage III with high blood lymphocyte level was better compared to those with low level, whereas there was no significant difference of OS between non-A subgroups. It was known that CD8+ effector cells often had an antitumor function in patients with NPC (Fogg M et al., 2013). A recent study demonstrated that cell therapy with EBV-specific autologous cytotoxic T lymphocytes (CTLs) might control disease progression in patients with NPC (Secondino et al., 2012). Another clinical study partly supported our results that obvious infiltration of $\mathrm{T}$ cell around neoplasm was associated with better prognosis of NPC patients in UICC stage III (He et al., 1999). Blood lymphocyte counting partially may reflect the infiltration of lymphocyte around tumor tissue, which explained our observations to a certain extent. There was much room for further studies on the interactions between sub-classifications of lymphocytes and prognosis of male NPC patients with blood group A.

NPC is characterized by a strong association to $\mathrm{EBV}$, thus giving rationale for viral antigens-targeted immunotherapy (Thompson et al., 2004). Moreover, recent studies observed that EBV-specific immunity enhanced by various strategies, including adoptive and active immunotherapy, could show anti-tumor effect significantly(Chua D et al., 2001; Straathof KC et al., 2003; Comoli P et al., 2004; Straathof KC et al., 2005). Nevertheless, most of the strategies were not mature enough for clinical treatment because of their lack of substantial data from clinical trial, from which patients usually could only get limited remission. Therefore, it is a promising way to promote the effect of EBV-targeted immunotherapy by identifying a special group of NPC patients through EBV-related clinical features. In our study, the association between EBV-induced immunity and prognosis has been revealed in male NPC patients with A blood group in certain UICC stage, which possibly provided a potential subgroup that might be sensitive to EBV-targeted immunotherapy.

In conclusion, our investigation suggested that $\mathrm{ABO}$ blood group status was not associated with the prognosis of patients with NPC in Chinese populations. Additionally, we identified a special subgroup of NPC patients that have male sex and A blood group, for whom high VcA-IgA level and high blood lymphocyte level might be correlated with a favorable prognosis in UICC stage II and UICC stage III, respectively. Moreover, we speculated that this special subgroup might be more sensitive to EBV-targeted immunotherapy. It is worthy of further investigation on the underlying mechanism. Besides, it is encouraged to investigate the roles of EBV DNA and classification of lymphocytes. Further studies on the genetic background of male NPC patients with blood group A are warranted.

\section{References}

Cai YL, Li J, Lu AY, et al (2013). Prognostic significance of serum anti-Epstein-Barr virus antibodies in nasopharyngeal carcinoma. Zhonghua Shi Yan He Lin Chuang Bing Du Xue Za Zhi, 27, 119-22

Cheng WM, Chan KH, Chen HL, et al (2002). Assessing the risk of nasopharyngeal carcinoma on the basis of EBV antibody spectrum. Int J Cancer, 97, 489-92.

Chien YC, Chen JY, Liu MY, et al (2001). Serologic markers of Epstein-Barr virus infection and nasopharyngeal carcinoma in Taiwanese men. $N$ Eng J Med, 345, 1877-82.

Chua D, Huang J, Zheng B, et al (2001). Adoptive transfer of autologous Epstein-Barr virus-specific cytotoxic $\mathrm{T}$ cells for nasopharyngeal carcinoma. Int J Cancer, 94, 73-80.

Comoli P, De Palma R, Siena S, et al (2004). Adoptive transfer of allogeneic Epstein-Barr virus (EBV)-specific cytotoxic $\mathrm{T}$ cells with in vitro antitumor activity boosts LMP2specific immune response in a patient with EBV-related nasopharyngeal carcinoma. Ann Oncol, 15, 113-7.

Fan H, Nicholls J, Chua D, et al (2004). Laboratory markers of tumor burden in nasopharyngeal carcinoma: a comparison of viral load and serologic tests for Epstein-Barr virus. Int J Cancer, 112, 1036-41.

Fogg M, Murphy JR, Lorch J, Posner M, Wang F (2013). Therapeutic targeting of regulatory $\mathrm{T}$ cells enhances tumor-specific CD8+ T cell responses in Epstein-Barr virus associated nasopharyngeal carcinoma. Virology, 441, 107-13.

Gao S, Worm J, Guldberg P, et al (2004). Genetic and epigenetic alterations of the blood group $\mathrm{ABO}$ gene in oral squamous cell carcinoma. Int J Cancer, 109, 230-7.

Gates MA, Wolpin BM, Cramer DW, Hankinson SE, Tworoger SS (2011). ABO blood group and incidence of epithelial ovarian cancer. Int J Cancer, 128, 482-6.

Han BL, Xu XY, Zhang CZ, et al (2012). Systematic review on Epstein-Barr Virus (EBV) DNA in diagnosis of nasopharyngeal carcinoma in Asian populations. Asian Pac J Cancer Prev, 13, 2577-81.

Hashim NAN, Ramzi NH, Velapasamy S, et al (2012). Identification of genetic and non-genetic risk factors for nasopharyngeal carcinoma in a southeast Asian population. Asian Pac J Cancer Prev, 13, 6005-10.

He C, Zhuang HG, Zhang WY (1999). The reaction and biological behavior of immune cells is associated with the prognosis of nasopharyngeal carcinoma in UICC stage III. Chinese J Pathol, 28, 115-8.

Henle G, Henle W (1976). Epstein-Barr virus-specific IgA serum antibodies as an outstanding feature of nasopharyngeal carcinoma. Int J Cancer, 17, 1-7.

Ho HC, Ng MH, Kwan HC, Chau JC (1976). Epstein-Barr virus specific IgA and IgG serum antibodies in nasopharyngeal carcinoma. Br J Cancer, 34, 655-60.

Hosoi E (2008). Biological and clinical aspects of ABO blood group system. J Med Invest, 55, 174-82.

Huang TR, Zhang SW, Chen WQ, et al (2012). Trends in nasopharyngeal carcinoma mortality in China, 1973-2005. Asian Pac J Cancer Prev, 13, 2495-502.

Iodice S, Maisonneuve P, Botteri E, Sandri MT, Lowenfels AB (2010). ABO blood group and cancer. Eur J Cancer, 46, 3345-50.

Jemal A, Bray F, Center MM, et al (2011). Global cancer statistics. CA Cancer J Clin, 61, 69-90.

Jia WH, Luo XY, Feng BJ, et al (2010). Traditional Cantonese diet and nasopharyngeal carcinoma risk: a large-scale casecontrol study in Guangdong, China. BMC Cancer, 10, 446.

Ji X, Zhang W, Xie C, et al (2011). Nasopharyngeal carcinoma risk by histologic type in central China: impact of smoking, alcohol and family history. Int J Cancer, 129, 724-32.

Joh HK, Cho E, Choueiri TK (2012). ABO blood group and risk 

of renal cell cancer. Cancer Epidemiol, 36, 528-32.

Low WK, Leong JL, Goh YH, Fong KW (2000). Diagnostic value of Ep-stein-Barr viral serology in nasopharyngeal carcinoma. Otolaryngol Head Neck Surg, 123, 505-7.

Ouyang PY, Su Z, Mao YP, Liu Q, Xie FY (2013). Prognostic value of $\mathrm{ABO}$ blood group in southern Chinese patients with established nasopharyngeal carcinoma. Br J Cancer advance online publication, $\mathbf{5 5 9}$.

Ozelius LJ, Kwiatkowski DJ, Schuback DE, et al (1992). A genetic linkage map of human chromosome 9q. Genomics, 14, 715-20.

Rickinson AB, Kieff E (2007) Epstein-Barr virus, $5^{\text {th }}$ ed. eds knipe, D.M.and howley, P.M. fields virology. Lippincott Williams \& Wilkins, Philadelphia, PA 2655-700.

Sam CK, Prasad U, Pathmanathan R (1989). Serological markers in the diagnosis of histopathological types of nasopharyngeal carcinoma. Eur J Surg Oncol, 15, 357-60.

Secondino S, Zecca M, Licitra L, et al (2012). T-cell therapy for EBV-associated nasopharyngeal carcinoma: preparative lymphodepleting chemotherapy does not improve clinical results. Ann Oncol, 23, 435-41.

Sheng L, Sun X, Zhang L, Su D (2013). ABO blood group and nasopharyngeal carcinoma risk in a population of Southeast China. Int J Cancer, 133, 893-7.

Straathof KC, Bollard CM, Popat U, et al (2005). Treatment of nasopharyngeal carcinoma with Epstein-Barr virus-specific T lymphocytes. Blood, 105, 1898-904.

Straathof KC, Bollard CM, Rooney CM, Heslop HE (2003). Immunotherapy for Epstein-Barr virus-associated cancers in children. Oncologist, 8, 83-98.

Thompson MP, Kurzrock R (2004). Epstein-Barr virus and cancer. Clin Cancer Res, 10, 803-21.

Tiwawech D, Srivatanakul P, Karaluk A, Ishida T (2003). Significance of plasma IgA and IgG antibodies to Epstein-Barr virus early and viral capsid antigens in Thai nasopharyngeal carcinoma. Asian Pac J Cancer Prev, 4, 113-8.

Wang Z, Liu L, Ji J, et al (2012). ABO Blood Group System and Gastric Cancer: A Case-Control Study and Meta-Analysis. In. J Mo. Sci, 13, 13308-21.

Wolpin BM, Chan AT, Hartge P, et al (2009). ABO blood group and the risk of pancreatic cancer. $J$ Natl Cancer Inst, 101, 424-31.

Zhang BL, He N, Huang YB et al (2014). ABO blood groups and risk of cancer: a systematic review and meta-analysis. Asian Pac J Cancer Prev, 15, 4643-50. 